\section{Addendum: A lysosomal switch triggers \\ proteostasis renewal in the immortal C.elegans germ lineage}

https://doi.org/10.1038/s41586-020-2108-0

Addendum to: Nature https://doi.org/10.1038/nature24620

Published online 22 November 2017

Check for updates

Jérôme Goudeau, Madhuja Samaddar, K. Adam Bohnert \&

Cynthia Kenyon

In this Letter, we described a shift in lysosome activity and mitochondrial metabolism that triggers the removal of protein aggregates in Caenorhabditis elegans oocytes when their maturation is stimulated by signals from sperm. An early step in this process is the acidification of lysosomes. We postulated that signals from sperm trigger lysosome acidification by stimulating the proteasomal destruction of the GLD-1 translational repressor, which in turn enables the synthesis of lysosomeacidifying V-ATPase subunits (Fig. 3 of our Letter). In support of this conclusion, the GFP::GLD-1-expressing transgene we used to visualize GLD-1 (axIs1498[Ppie-1::gfp::gld-1::gld-13'UTR, unc-119(+)] ${ }^{1}$ resulted in GFP expression in proximal oocytes of females (which lack sperm) but not in those of hermaphrodites (which have sperm) (Fig. 3 of our Letter). After our Letter was published, D. Greenstein informed us that this GLD-1 transgene probably produced a misleading GFP signal, because neither a different GLD-1::GFP transgene nor a GLD-1 antibody produced a detectable signal in the proximal oocytes of females (see ref. ${ }^{2}$ for data and a discussion of these transgenes). His results prompted us to take an approach that was less likely to generate artefacts; namely, to tag the endogenous gld-1 gene with GFP using CRISPR-Cas9 technology. Using this strain, we were also unable to detect a GLD-1::GFP signal in the proximal oocytes of females (see Fig. 1, Supplementary Fig. 1 and Supplementary Methods of this Addendum). This suggests that the proteasomal activity that degrades GLD-1 in the proximal oocytes of hermaphrodites (further explored by ref. ${ }^{2}$ ) may also degrade GLD-1 in the proximal oocytes of females, and something other than GLD-1 may prevent lysosomes from being acidified in the absence of sperm. This mechanism remains unresolved, and is under investigation in our laboratory. Figure 2 of this Amendment describes our revised model, in which the fundamental conclusions of the study remain intact: signals from sperm trigger the destruction of protein aggregates in female oocytes by stimulating lysosome acidification (see Figs. 1 and 2 of our Letter). Lysosome acidification enables mitochondria to transit from what appears to be a poised state (with a high membrane potential) to an active state (with a lower, hermaphrodite-like membrane potential). This shift in mitochondrial physiology is required for the acidified lysosomes to engulf protein aggregates (Fig. 4 of our Letter), probably by the process of microautophagy (Fig. 2 of our Letter).

We thank D. Greenstein for bringing this matter to our attention. AuthorsJ.G. and M.S., both members of the Kenyon laboratory at Calico Life Sciences, carried out the analyses described in this Amendment and

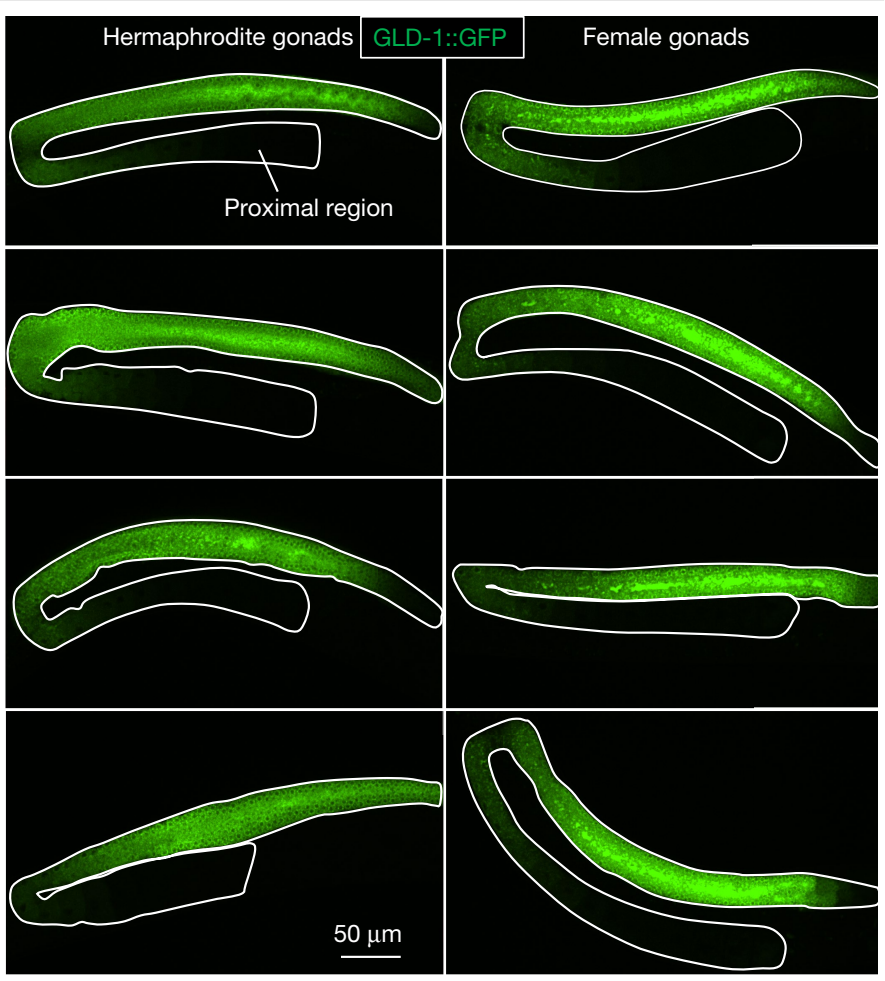

Fig. 1|A GLD-1::GFP fusion protein expressed from the endogenously tagged gld-1 gene is present in distal but not proximal oocytes in both females and hermaphrodites. Shown are four females and hermaphrodites from a total of 10 females and 12 hermaphrodites examined. The remaining animals are shown in Supplementary Fig. 1. Animals carrying endogenously tagged GLD-1::GFP were grown from hatching on bacteria expressing fem-1 dsRNA (females) or empty-vector control (hermaphrodites) at $20^{\circ} \mathrm{C}$. Genotype: gld-1(syb798) I; unc-119(ed3) III; ieSi38 [sun-1p::TIR1::mRuby::sun-13'UTR+Cbr-unc-119(+)] IV. The endogenous, functional gld-1(syb798) allele contains a GFP::Degron tag inserted using CRISPR-Cas9. The strain, PHX798, was made for the Kenyon laboratory by SunyBiotech.

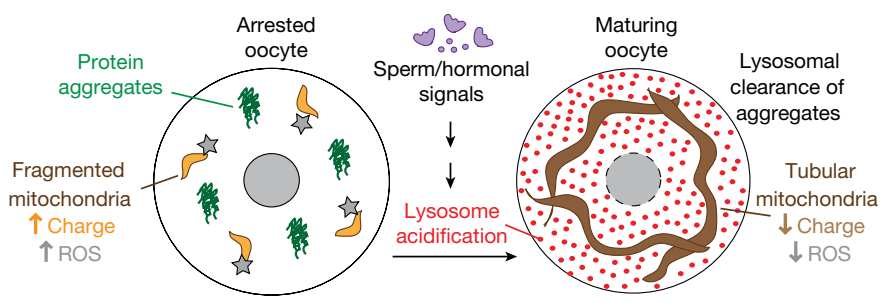

Fig. 2 | Revised model. Compare with Fig. 5 of our Letter.

contributed equally.J.G. designed the endogenously tagged gld-1::gfp strain in silico, and performed the experiments described in Fig. 1 of this Amendment. M.S. imaged other animals independently, with similar findings. M.S. also examined several GLD-1 reporter strains sent to us by D. Greenstein and confirmed the observations of ref. ${ }^{2}$. As part of our ongoing experiments, J.G. and M.S. have also repeated and confirmed our published observations regarding lysosomal acidification, mitochondrial membrane potential and protein aggregation in hermaphrodites and females. We thank R. Kerr for automated image analysis.

Supplementary Information is available in the online version of this Amendment.

Merritt, C., Rasoloson, D., Ko, D. \& Seydoux, G. 3' UTRs are the primary regulators of gene expression in the C. elegans germline. Curr. Biol. 18, 1476-1482 (2008).

2. Spike, C. A., Huelgas-Morales, G., Tsukamoto, T. \& Greenstein, D. Multiple mechanisms inactivate the LIN-41 RNA-binding protein to ensure a robust oocyte-to-embryo transition in Caenorhabditis elegans. Genetics 210, 1011-1037 (2018). 